\title{
Estresse e Suporte Social na Infầncia e Adolescência relacionados com Sintomas Depressivos em Idosos
}

\author{
Stress and Social Support in Childhood and Adolescence \\ Related with Depression Symptoms in the Elderly
}

\author{
Ana Paula Fabrino Bretas Cupertino ${ }^{a}$, Beatriz Helena Domingos Oliveira ${ }^{b *}$, \\ Danielle Viveiros Guedes ${ }^{\mathrm{a}}$, Evelyne Rosa Coelho ${ }^{\mathrm{a}}$, Roberta Saramella Milano, \\ Jacqueline Silva Rubac ${ }^{\text {a }}$, Sumaya Hallack Sarkis ${ }^{\mathrm{a}}$ \\ ${ }^{a}$ Universidade Federal de Juiz de Fora, Juiz de Fora, Brasil, ${ }^{b}$ Universidade Federal de Campinas, Campinas, Brasil
}

\begin{abstract}
Resumo
Considerando o envelhecimento como período ligado às influências sócio-históricas e experiências ocorridas durante o desenvolvimento, torna-se importante investigar variáveis de outros períodos do curso da vida que possam estar implicadas na saúde dos idosos. Assim, o objetivo deste estudo foi avaliar a relação entre características do suporte social e estresse na infância/adolescência com sintomas depressivos na velhice. Utilizou-se a escala CES-D (Radloff, 1977) e a escala de suporte social e estresse na infância/adolescência do Davis Longitudinal Study on Aging (Aldwin, Sutton, Chiara, \& Spiro, III, 1996). A amostra consistiu de 956 idosos com idade entre 60 e 103 anos $(\mathrm{M}=72,4 ; \mathrm{DP}=8,2)$. Foram encontradas relações significativas entre estressores e disciplina recompensatória com sintomas depressivos, o que se atribui a características nãonormativas destas variáveis para esta coorte.

Palavras-chave: Estresse; suporte social; sintomas depressivos; envelhecimento.
\end{abstract}

\begin{abstract}
Considering the process of aging as a period connected to social and historical influences, and life experiences that occur during development, it's significant to investigate the variables of other periods from the course of life, which could be implicated in the health of elders. The purpose of this study, however, was to assess the relation amongst social support characteristics and stress during the childhood/adolescence period, and depression symptoms of the elderly. The CES-D scale (Radloff, 1977) and the social support and stress in childhood/adolescence scale from Davis Longitudinal Study on Aging (Aldwin, Sutton, Chiara, \& Spiro, III, 1996) were used. The sample consisted of 956 elderly between 60 and 103 years old $(\mathrm{M}=72,4 ; \mathrm{SD}=8,2)$. Significant connections had been found between stressors and compensatory discipline with depression symptoms, which has been attributed to non-normative characteristics of such variables for the present cohort.

Keywords: Stress; social support; depression symptoms; aging.
\end{abstract}

O envelhecimento é um processo caracterizado, dentre outros aspectos, pela multidirecionalidade, multidimensionalidade, plasticidade e capacidade de resiliência (Staudinger, Marsiske \& Baltes, 1995). Considerando a multidirecionalidade intrínseca ao envelhecimento humano, Rowe e Kahn (1998) propõem três perspectivas didáticas para examinar os processos do envelhecimento: normal, patológico ou saudável. O envelhecimento normal representa o processo natural de desenvolvimento no qual o indivíduo consegue se adaptar às alterações típicas e inevitáveis do envelhecimento (Neri, 2001). Por outro

\footnotetext{
* Endereço para correspondência: Rua da Passagem A, n. 08, Linhares, Juiz de Fora, MG, 36.060-000. E-mail: izjf2004@yahoo.com.br

Agradecemos ao Programa Especial de estímulo à fixação de doutores - PROFIX/ $\mathrm{CNPq}$, aos bolsistas de Apoio Técnico e de Iniciação Científica do CNPq durante o período de 2001 a 2004, bem como aos alunos de graduação do curso de Psicologia matriculados na disciplina Treino de Pesquisa entre 2001 e 2003.
}

lado, o envelhecimento patológico caracteriza-se por prejuízos graves, irreversíveis e cumulativos ao funcionamento individual, além de uma descontinuidade do desenvolvimento. Por último, o envelhecimento saudável, também chamado de ótimo e referenciado como um ideal sociocultural de excelente qualidade de vida, manifestase por baixo risco de doenças e incapacidades, boa funcionalidade física e mental e engajamento ativo com a vida (Neri, 2002).

A variabilidade no perfil de envelhecimento emocional tem sido alvo de muitos estudos buscando compreender a relação, por exemplo, entre depressão e idade. No Brasil, dados epidemiológicos apresentam uma prevalência variando ao redor de $15 \%$ de sintomas depressivos e $4 \%$ de depressão maior (Stoppe Jr. \& Neto, 1999) entre idosos. Entretanto, não há um consenso entre os estudos de prevalência de depressão entre idosos devido ao uso de diferentes metodologias de estudo e definições do termo (Gazalle, Lima, Tavares \& 
Hallal, 2004; Miguel Filho \& Almeida, 2000). Sendo assim, a relação entre depressão e idade não é simples e casual, existindo, portanto, variáveis que podem influenciar a variabilidade do perfil emocional no envelhecimento. Diante deste contex to da complexidade da compreensão da relação entre idade e depressão, além de identificar se os idosos estão deprimidos, torna-se fundamental explorar fatores relacionados com a depressão entre idosos. Considerando que a depressão em idosos pressupõe uma compreensão multifatorial, dada a presença de características específicas na clínica e no tratamento (Gazalle et al., 2004), as variáveis psicossociais se apresentam como fatores relacionados com a presença de depressão na idade avançada (Blazer, Hughes \& George, 1987; Lafer, Almeida, Fraguas Jr. \& Miguel Filho, 2001; Snowdon, 2002; Stoppe Jr. \& Neto, 1999).

Uma outra hipótese explicativa da presença de sintomas depressivos na velhice são as variáveis presentes na infância ou adolescência enquanto exercendo um efeito em longo prazo na saúde emocional dos idosos. Ou seja, no curso da vida, muitos indivíduos crescem e se desenvolvem em contextos e situações que repercutem na saúde psicológica (Cecconello \& Koller, 2000). Os possíveis eventos estressores negativos na infância, tais como brigas e/ou separação dos pais, vivência escolar, morte na família, exigências e/ou rejeição de colegas, disciplina dos pais, hospitalização, doença e mudança de casa (Lipp, 2000) podem estar relacionados com sintomas depressivos em outros momentos do curso da vida, resultando em danos para o desenvolvimento global do indivíduo. Em uma amostra americana, Aldwin, Spiro III, Levenson e Cupertino (2001) encontraram que o perfil socioeconômico na infância e adolescência estaria relacionado ao perfil de morbi-mortalidade no envelhecimento. Concluindo, variáveis da infância e adolescência podem desempenhar um papel fundamental na compreensão da saúde física e psicológica dos indivíduos em períodos posteriores da vida, especificamente na velhice.

Por outro lado, variáveis da infância e adolescência podem ter o efeito protetor em longo prazo na saúde emocional de idosos. Por exemplo, a presença de variáveis protetoras, tais como suporte social - representado pela existência ou disponibilidade de uma rede de suporte social, com que a criança e o adolescente possam contar, se sentirem importantes, valorizados e amados - pode amenizar a experiência do estresse e os efeitos do mesmo na saúde emocional de idosos (Goldstein, 1995).

Sendo assim, o objetivo deste artigo é investigar a correlação entre variáveis presentes no início do curso da vida com a saúde emocional de idosos. Especificamente, serão examinadas características da correlação entre eventos estressantes e de suporte social na infância e adolescência com a variabilidade de sintomas depressivos na velhice.

\section{Método}

\section{Amostra}

A amostra consiste de 956 idosos com idade variando entre 60 e 103 anos $(\mathrm{M}=72,4 ; \mathrm{DP}=8,2)$. Ainda com relação às características socioeconômicas da amostra, $72 \%$ são mulheres, $32 \%$ têm o primário completo $(\mathrm{M}=6,97$; $\mathrm{DP}=4,45)$ e apenas $13 \%$ moram sozinhos.

\section{Instrumentos}

Para avaliar sintomas depressivos utilizou-se a escala de rastreamento CES-D (Center Epidemiologic Survey Depression) desenvolvida por Radloff (1977) com a finalidade de detectar sintomas depressivos em populações adultas, também utilizada em uma amostra de universitários por Silveira e Jorge (2000), e de idosos por Tavares (2004). Nesta escala investiga-se a freqüência de ocorrência de 20 sintomas depressivos na última semana, totalizando a soma de um escore entre 0 e 60.

Com relação às variáveis estresse e suporte social na infância e adolescência, aplicou-se a versão cross-traduzida para o inglês e português por Cupertino (2001) da escala utilizada por Aldwin, Cupertino, Levenson e Spiro III (1998) no Davis Longitudinal Study on Aging(DLS). A escala possui 15 itens característicos da rede de suporte social e de estressores durante a infância e a adolescência. Estes itens são dicotômicos (sim/não), e em caso de resposta positiva, pede-se ao entrevistado que indique se o acontecimento ocorreu entre as seguintes faixas etárias: 0-5 anos, 5-12 anos e de 12-19 anos.

\section{Suporte social}

Esta parte da escala é composta por quatro itens descrevendo a qualidade e a quantidade da rede de suporte social (sentir-se próximo de algum parente, de algum outro adulto, de algum irmão em particular, e de um amigo). Os quatro itens foram somados, totalizando em uma escala contínua que varia entre 0 (sem rede de suporte social na infância e adolescência) e 4 (rede de suporte ampla).

\section{Estressores}

Para avaliar os estressores foram investigados 11 tipos de estressores na infância e adolescência (problemas financeiros dos pais, ficar separado dos pais por mais de um mês, separação dos pais, perder o pai ou a mãe, perder algum parente próximo, ficar severamente doente, ficar de quarentena, ter sofrido algum acidente, irmão gravemente doente, mudar de residência na mesma cidade e mudar para outra cidade). Os itens foram somados, resultando em uma escala que varia entre $\mathrm{O}$ (nenhum estressor na infância e adolescência) e 11 (infância e adolescência marcadas por variedade de estressores).

Para investigar as disciplinas foram avaliadas duas dimensões: características da punição e recompensa dadas pelos pais na infância e adolescência. Ou seja, os participantes do estudo foram perguntados sobre como os pais reagiam frente a algo que faziam de errado, bem como quando faziam algo positivo durante a infância e adolescência.

1. Punição: esta variável foi explorada em quatro categorias não excludentes entre si. A primeira (sem disciplina) engloba os itens: nunca foi disciplinado e ignorando. A segunda consiste na presença de algum tipo 
de punição física ou não (espancando, batendo, batendo com cinto, batendo com varas, e chutando). A terceira categoria investiga a presença de castigo ou não, e a última categoria investiga a presença de repreensão verbal na disciplina dada pelos pais durante a infância e a adolescência.

2. Recompensa: além das disciplinas, foi também investigado o que os pais faziam quando ficavam felizes com algo que o indivíduo fez durante sua infância e adolescência. Esta variável engloba três categorias de respostas. A primeira (sem demonstração) composta pelo item: eles quase nunca ficavam felizes ou demonstravam; a segunda (demonstração verbal), pelo item demonstravam verbalmente; e a terceira (com recompensa) pelos seguintes itens: faziam algum agrado especial e davam alguma recompensa.

\section{Lembranças de desempenho}

Abordou-se as lembranças positivas da infância e adolescência através de seis itens dicotômicos: vocêfoi considerado levado quando criança, considerado inteligente, considerado atleta, considerado popular/ cheio de amigos, participou de algum grupo (escoteiro, igreja, dentre outros) e destacou-se em algo que fez enquanto crescia (notas, música, hobbies, etc.). O escore total desta variável tem como mínimo 0 e máximo 6 .

Avaliação da infância e adolescência: por último, era pedido aos entrevistados que atribuíssem uma nota de 1 a 10 para o período, sendo 1 péssimo e 10 ótimo.

\section{Procedimento}

As escalas foram aplicadas em todos os idosos da amostra do Estudo dos Processos de Envelhecimento Saudável PENSA (Cupertino, Neri, Silva, Banhato, \& Novaes, 2004). A aplicação destas escalas foi realizada por entrevistadores treinados (graduandos de psicologia) e teve uma duração média de 15 minutos.

\section{Resultados}

Considerando o suporte social na infância e adolescência (Tabela 1), 60\% da amostra tiveram algum suporte familiar, $55 \%$ um irmão mais próximo, $54 \%$ um amigo em particular na infância, e $33 \%$ suporte de um outro adulto. Somando o total da rede de suporte social dos idosos na infância e adolescência, identificou-se uma variação entre os idosos, desde aqueles que relataram não ter ninguém na sua rede de suporte social ( $14 \%$ tiraram zero na soma) até idosos que relataram uma rede de suporte social ampla $(\mathrm{M}=2,0 ; \mathrm{DP}=1,2)$.

Quanto aos estressores experienciados na infância e adolescência (Tabela 2), os mais citados pelos idosos foram: problemas financeiros (56\%), perda de alguma pessoa próxima $(47 \%)$, mudança de residência $(46 \%)$ e ter perdido um dos pais durante o período (29\%). Com relação ao total de estressores, apenas $5 \%$ da amostra não teve nenhum acontecimento estressante no período $(\mathrm{M}=2,9 ; \mathrm{DP}=1,6)$.

Com relação ao primeiro aspecto da disciplina recebida dos pais durante a infância e adolescência, observou-se a seguinte distribuição entre as categorias: $40 \%$ relataram ter recebido punição física dos pais durante a infância e adolescência, $40 \%$ punição verbal, $15 \%$ algum tipo de castigo e $5 \%$ relataram não ter recebido punição dos pais.

Por outro lado, observou-se que as recompensas recebidas dos pais, quando realizado um comportamento positivo,

Tabela 2

Estressores na Infância e Adolescência

\begin{tabular}{lcc}
\hline Acontecimentos estressantes & $\mathrm{f}$ & \%* \\
\hline Problemas financeiros & 534 & 56 \\
Perda de pessoa próxima & 444 & 47 \\
Mudar de residência & 440 & 46 \\
Mudar para outra cidade & 356 & 38 \\
Ser separado dos pais & 289 & 31 \\
Perda de um dos pais & 272 & 29 \\
Sofrer algum acidente grave & 248 & 26 \\
Ficar severamente doente & 225 & 24 \\
Irmão com doença grave & 174 & 18 \\
Ficar de quarentena & 107 & 11 \\
Separação dos pais & 58 & 6 \\
\hline
\end{tabular}

Nota. $*$ O total das porcentagens excede $100 \%$, uma vez que os entrevistados não foram solicitados a apontar o evento mais estressante, mas todos os que ocorreram na infância e adolescência.

Tabela 1

Freqüências do Suporte Social, Lembranças e Atividades de Destaque

\begin{tabular}{|c|c|c|c|c|c|c|c|c|}
\hline Suporte social & $\mathrm{f}$ & $\% *$ & Lembranças & $\mathrm{f}$ & $\%$ * & Atividades de destaque & $\mathrm{f}$ & $\% *$ \\
\hline Suporte familiar & 572 & 60 & Inteligente & 532 & 56 & Boas notas & 154 & 37 \\
\hline Irmão mais próximo & 524 & 55 & Popular/ cheio de amigos & 525 & 55 & Esportes & 70 & 16 \\
\hline Amigo próximo & 516 & 54 & Participação de grupo & 390 & 40 & Música & 47 & 11 \\
\hline \multirow[t]{2}{*}{ Outro adulto } & 316 & 33 & Levados & 315 & 33 & Escola & 31 & 7 \\
\hline & & & Atletas & 148 & 16 & Hobbies & 11 & 3 \\
\hline
\end{tabular}

Nota. * O total das porcentagens das colunas podem ser diferentes de $100 \%$ devido ao fato de que os participantes tinham opção de responder nenhum (a) ou mais de uma opção para os três itens (suporte social, lembranças e atividades de destaques). 
foram principalmente verbais (53\%), seguidas de não reconhecimento (34\%). Apenas $11 \%$ dos idosos relataram ter recebido algum tipo de recompensa dos pais na infância e adolescência quando faziam algo positivo.

Ainda com relação à disciplina recebida dos pais, por meio da análise de variância entre indivíduos que receberam e não receberam punição física e sintomas depressivos na velhice, foi encontrada uma tendência à presença de sintomas depressivos entre os idosos que relataram ter recebido punição física dos pais na infância e adolescência $\left(\mathrm{F}_{(\mathrm{X}, \mathrm{Y})}=\mathrm{Z} ; \mathrm{p} \leq 0,05\right)$. Por outro lado, através da mesma análise, entre as categorias de quem recebeu recompensa dos pais e não recebeu, e sintomas depressivos, foi encontrada uma relação significativa, observando-se que os idosos que recebiam alguma recompensa apresentaram menores escores de sintomas depressivos $\left(\mathrm{F}_{(\mathrm{X}, \mathrm{Y})}=\mathrm{Z} ; \mathrm{p} \leq \mathrm{0,01}\right)$.

Utilizou-se também o teste Chi-quadrado para avaliar a relação entre os que receberam recompensa ou não (Tabela 3) e os que receberam punição física ou não (Tabela 4), durante a infância e adolescência com as três categorias de sintomas depressivos na velhice (sem depressão, moderadamente deprimido e severamente deprimido). Primeiramente, categorizou-se os escores de sintomas depressivos em três grupos (de 0 a 14 - sem depressão; de 15 a 21 - moderadamente deprimidos; 22 ou mais - severamente deprimidos). Com relação à punição física, não houve relação estatisticamente significativa, $\mathrm{X}^{2}(2)=3,83, \mathrm{p}=0,15$, porém, observou-se uma maior prevalência dos indivíduos que não receberam este tipo de disciplina na categoria 1 (sem depressão). Quanto à variável recompensa, observou-se uma relação estatisticamente significativa $\mathrm{X}^{2}(2)=11,89, \mathrm{p} \leq 0,01$, ou seja, a maioria dos idosos que relatou ter recebido recompensa dos pais na infância e adolescência quando fazia algo positivo apresentou baixos escores, estando na categoria 1 de sintomas depressivos na velhice.

Em síntese, estas análises entre disciplina e recompensa recebida na infância e adolescência com sintomas depressivos na velhice apontam para o fato de que ter recebido recompensa mostrou um efeito em longo prazo nos sintomas depressivos, enquanto ter recebido punição física não.

Tabela 3

Teste Chi-Quadrado das Variáveis Depressão e Recompensa

\begin{tabular}{|c|c|c|c|c|c|}
\hline & $f$ & $\%$ & $\begin{array}{c}\text { Sem } \\
\text { depressão - \% } \\
\end{array}$ & $\begin{array}{c}\text { Moderadamente } \\
\text { deprimido - \% }\end{array}$ & $\begin{array}{c}\text { Severamente } \\
\text { deprimido - \% }\end{array}$ \\
\hline Com recompensa & 582 & 64 & 51 & 7 & 6 \\
\hline Sem recompensa & 324 & 36 & 25 & 6 & 5 \\
\hline \multicolumn{6}{|l|}{ Total de depressão } \\
\hline \multirow[t]{2}{*}{ em categorias } & 76 & 13 & 11 & & \\
\hline & & \multicolumn{2}{|r|}{ Valor } & gl & Significância \\
\hline \multicolumn{2}{|c|}{ Pearson chi-square } & \multicolumn{2}{|r|}{$11,892^{\mathrm{a}}$} & 2 & 0,003 \\
\hline \multicolumn{2}{|c|}{ Probabilidade de relação } & \multicolumn{2}{|r|}{11,622} & 2 & 0,003 \\
\hline \multicolumn{2}{|c|}{ Associação Linear-by-linear } & \multicolumn{2}{|r|}{11,356} & 1 & 0,001 \\
\hline \multicolumn{2}{|c|}{ Número de casos válidos } & \multicolumn{2}{|r|}{906} & & \\
\hline
\end{tabular}

Nota. 2 células (33,3\%) esperaram resultado menor que 5 . O resultado mínimo esperado 4,19.

Tabela 4

Teste Chi-Quadrado das Variáveis Depressão e Punição

\begin{tabular}{|c|c|c|c|c|c|}
\hline & $\mathrm{f}$ & $\%$ & $\begin{array}{c}\text { Sem } \\
\text { depressão - \% }\end{array}$ & $\begin{array}{c}\text { Moderadamente } \\
\text { deprimido - \% }\end{array}$ & $\begin{array}{r}\text { Severamente } \\
\text { deprimido - \% }\end{array}$ \\
\hline Com punição & 839 & 95 & 73 & 12 & 10 \\
\hline Sem punição & 40 & 5 & 4 & 0,2 & 0,2 \\
\hline \multirow[t]{2}{*}{ em categorias } & 77 & 12 & 11 & & \\
\hline & & \multicolumn{2}{|r|}{ Valor } & gl & Significância \\
\hline \multicolumn{2}{|c|}{ Pearson chi-square } & \multicolumn{2}{|r|}{$3,833^{\mathrm{a}}$} & 2 & 0,147 \\
\hline \multicolumn{2}{|c|}{ Probabilidade de relação } & \multicolumn{2}{|r|}{4,555} & 2 & 0,103 \\
\hline \multicolumn{2}{|c|}{ Associação Linear-by-linear } & \multicolumn{2}{|r|}{3,185} & 1 & 0,074 \\
\hline \multicolumn{2}{|c|}{ Número de casos válidos } & \multicolumn{2}{|r|}{879} & & \\
\hline
\end{tabular}

Nota. 2 células (33,3\%) esperaram resultado menor que 5 . O resultado mínimo esperado 4,19. 
Em relação às lembranças da infância e adolescência, como apresentado anteriormente (Tabela 1), $56 \%$ da amostra afirmaram ter sido considerados inteligentes, $55 \%$ populares, $40 \%$ participaram de algum grupo, $33 \%$ eram considerados levados e $16 \%$ atletas. Além disso, $40 \%$ relataram ter realizado alguma atividade em que se destacou durante o período, tais como, boas notas (37\%), esportes (16\%) e música (11\%). Quanto ao total de lembranças, observou-se que os escores variaram entre 0 e 6 , sendo que $48 \%$ da amostra apresentaram escores acima de 3 , indicando uma representação satisfatória das suas lembranças de desempenho na infância e adolescência $(\mathrm{M}=2,4 ; \mathrm{DP}=1,6)$.

Apesar da elevada presença de estressores para a maioria da amostra, os idosos fizeram uma avaliação positiva de sua infância e adolescência. Observou-se que na avaliação quantitativa a maioria dos idosos $(76 \%)$ avaliou sua infância com notas entre 8 e $10(\mathrm{M}=8,27 ; \mathrm{DP}=2,14)$, e apenas $5 \%$ com notas entre 1 e 4 .

Quanto aos sintomas depressivos atuais (Tabela 5), observou-se que os escores variaram entre 0 e 53, com valor de média baixo ( $\mathrm{M}=10,12 ; \mathrm{DP}=8,7)$, e ainda, que $10 \%$ da amostra foi identificada como severamente deprimida, $13 \%$ como moderadamente deprimida e $77 \%$ apresentaram escores entre 0 e 14, não estando, portanto, deprimida.

Tabela 5

Escores de Sintomas Depressivos em Categorias

\begin{tabular}{lcc}
\hline Categorias de sintomas depressivos & f & \%* \\
\hline 1- Sem depressão (O a 14) & 736 & 77 \\
2- Moderadamente deprimido (15 a 2 1) & 115 & 12 \\
3- Severamente deprimido (22 ou mais) & 105 & 11 \\
\hline
\end{tabular}

Examinando a correlação de Pearson entre as variáveis estudadas (Tabela 6), três aspectos merecem destaque. Primeiro, a característica da família brasileira representada nesta coorte histórica pela correlação positiva $(r=0,12)$ entre alta presença de suporte social relacionada com a presença de estressores na infância e adolescência. Um segundo aspecto refere-se à alta nota atribuída para a infância pela maioria dos idosos, estando relacionada, conforme esperado, com menos estresse $(r=-0,14)$ e maior presença de uma rede de suporte social $(r=0,17)$ na infância e adolescência. Por último, sintomas depressivos na velhice estiveram relacionados significativamente com maior presença de estressores $(r=0,08)$ e menor reconhecimento dos pais $(r=-0,12)$, enquanto presença de suporte social não apresentou relação significativa com a saúde emocional na velhice. $\mathrm{Na}$ direção esperada, a nota atribuída à infância esteve negativamente associada com sintomas depressivos $(r=-0,15)$, o que indica que aqueles que avaliaram a infância mais negativamente apresentaram mais sintomas depressivos.

Resumindo, verificou-se que para esta coorte histórica, estresse e recompensa na infância e adolescência tendem a ter um efeito em longo prazo relacionado com sintomas depressivos na velhice, contudo, o mesmo não se aplica para punição física e suporte social.

\section{Discussão}

As relações entre as variáveis investigadas apresentaram-se, em sua maioria, de forma estatisticamente significativa e na direção esperada. Porém, também houve variáveis que não corroboraram algumas das hipóteses iniciais.

Abordando a variável suporte social, observou-se que houve uma predominância da rede de suporte familiar, o que talvez se deva a uma característica desta coorte-histórica no que se refere à valorização das relações familiares. Ao contrário do esperado, suporte social e sintomas depressivos não apresentaram correlação significativa, o que aponta para o fato de que ter tido uma rede de suporte familiar ampla na infância não implicou em menor nem em maior número de sintomas depressivos na velhice. Uma possível explicação para o fato remete-se ao instrumento utilizado para investigar suporte social, já que este avalia o

Tabela 6

Correlação das Variáveis

\begin{tabular}{|c|c|c|c|c|c|c|c|c|}
\hline & 1 & 2 & 3 & 4 & 5 & 6 & 7 & 8 \\
\hline \multicolumn{9}{|l|}{1 - Idade } \\
\hline 2 - Suporte Social & $-0,07^{*}$ & & & & & & & \\
\hline 3 -Estressores & $-0,14^{* * *}$ & $0,15 * * *$ & & & & & & \\
\hline 4-Punição Física & $-0,04$ & 0,02 & 0,06 & & & & & \\
\hline 5 -Reconhecimento & 0,07 * & 0,11 ** & $-0,07 *$ & 0,06 & & & & \\
\hline 6 - Lembranças & $-0,07$ * & 0,32 ***** & $0,10^{* * *}$ & 0,07 * & 0,15 **** & & & \\
\hline $7-$ Nota & $0,08^{*}$ & 0,17 **** & $-0,13^{* * * * *}$ & $-0,05$ & 0,26 米米 & $0,22 * * * *$ & & \\
\hline $8-$ Sintomas depressivos & $-0,07^{*}$ & $-0,01$ & $0,08^{*} *$ & 0,06 & $-0,12$ * $* *$ & $-0,07^{*}$ & $-0,15^{* * * * *}$ & \\
\hline
\end{tabular}

Notas. **** $\mathrm{p} \leq 0,001$; ** $\mathrm{p} \leq 0,01 ;{ }^{*} \mathrm{p} \leq 0,05$ 
aspecto quantitativo da variável, não sendo talvez sensível o suficiente para analisar uma complexa relação como a do suporte social na infância e adolescência e sintomas depressivos na velhice, pelo fato de não abordar o aspecto qualitativo. Por outro lado, estes sintomas podem ter surgido em decorrência de fatores mais recentes, os quais influenciariam mais intensamente o estado emocional desses idosos.

Quanto à variável estressores, a maioria da amostra relatou ter experienciado acontecimentos estressantes, o que poderia também estar relacionado a uma característica desta coorte histórica, uma vez que o evento mais citado foi problemas financeiros, um aspecto do contex to econômico da época. Observou-se ainda que quanto maior o suporte social na infância e adolescência, maior foi o escore total de estressores, o que talvez tenha sido influenciado pelo perfil familiar desta coorte-histórica em que a família, por ser muito simbiótica e misturada, implica em uma não consideração dos aspectos individuais de seus membros (Mello, 1997 citado por Aquino \& Cabral, 2002).

Com relação às lembranças de desempenho, a maioria da amostra teve uma representação satisfatória desta variável, podendo-se atribuir este resultado às influências das experiências vividas e da sabedoria adquirida, o que implicaria em um menor rigor na avaliação das atividades e papéis desempenhados. Observou-se ainda que os idosos com maiores escores de lembranças de desempenho apresentaram menores escores de sintomas depressivos, podendo-se considerar, assim, que as recordações têm um papel na construção da auto-estima atual dos indivíduos, o que reduziria oscilações do estado de humor, ou seja, boas lembranças da infância podem ter um efeito minimizador sobre sintomas depressivos na velhice.

Além desta representação satisfatória das lembranças, a maioria dos idosos avaliou sua infância e adolescência positivamente, fato visualizado nas altas notas atribuídas para o período, podendo-se inferir que na velhice lembra-se da infância e adolescência com satisfação. Tal fato talvez se deva a um viés de falsa memória ou mesmo à verdade, ou seja, a infância e adolescência foram marcadas por momentos e vivências positivas. Observou-se ainda que quanto maior o suporte social, maior foi a nota atribuída, o que talvez aponte para a influência da transcendência, enquanto fator que promove um menor investimento em questões e acontecimentos interpessoais e materiais (Levenson, Aldwin \& Cupertino, 2001). Dessa forma, mesmo tendo vivenciado diversos eventos negativos estressantes, os idosos tendem a basear sua avaliação do período no aspecto positivo do suporte social, como ter um irmão próximo e um amigo íntimo, redes de suportes apontadas pela maioria da amostra. Verificou-se também que quanto maior a nota atribuída, menores foram os escores de sintomas depressivos destes idosos, demonstrando uma possível influência dos aspectos positivos de outros períodos do curso da vida sobre a trajetória atual de desenvolvimento.

Considerando as características da variável disciplina recebida dos pais, observou-se que $40 \%$ dos entrevistados relataram ter recebido punição física, enquanto que, quando emitido um comportamento aprovado, $34 \%$ não eram recompensados. Observou-se que os idosos que receberam recompensas durante a infância e adolescência quando emitiam um comportamento positivo apresentaram menores escores para sintomas depressivos na velhice. Contudo, não houve relação significativa entre punição física e sintomas depressivos, apontando para o fato de que ter recebido recompensa mostrou um efeito em longo prazo nos sintomas depressivos, enquanto ter recebido punição física, não. Uma explicação possível seria a de que para esta coorte histórica ser punido fisicamente era normativo, enquanto receber recompensa, não. Sabe-se que os eventos não-normativos são os que demandam maior adaptação dos indivíduos, podendo assim ter maiores implicações para o desenvolvimento (Neri, 1997).

Ressalta-se ainda, outras observações relacionadas à variável dependente - sintomas depressivos na velhice. Os idosos com maiores escores de total de estressores apresentaram maiores escores de sintomas depressivos, o que remete ao conceito de capacidade de resiliência. Segundo Cecconello e Koller (2000), observa-se que entre crianças que experienciam situações de risco em comum, nem todas apresentam dificuldades no desenvolvimento conseqüente. Algumas delas conseguem adaptar-se e superar essas situações, sendo assim mais resilientes, isto é, recuperando e mantendo o comportamento adaptativo quando ameaçadas por eventos estressantes (Garmezi, 1991 citado por Staudinger et al., 1995). Desta forma, a capacidade de resiliência pode estar entre as possíveis explicações para o fato de eventos estressantes na infância não necessariamente determinarem prejuízos no desenvolvimento posterior, como por exemplo, o surgimento de sintomas depressivos na velhice. Outra possível explicação seria a influência de aspectos sócio-históricos da amostra, pois sabe-se que cada coorte histórica experiencia determinados eventos que têm influência sobre a trajetória de desenvolvimento dos indivíduos. Ainda quanto à relação significativa entre estressores e sintomas depressivos, podese também inferir uma influência dos tipos de estressores mais vivenciados pela amostra durante a infância e adolescência, como perda de uma pessoa próxima e problemas financeiros, que consistem em eventos inesperados, os quais podem ser considerados como fatores que requerem um grande esforço adaptativo e estão sob pouca controlabilidade do indivíduo (Fortes \& Neri, 2004).

Em adição, conforme esperado, houve uma correlação negativa entre idade e sintomas depressivos, o que aponta para o fato de que envelhecer implica em um equilíbrio entre perdas e ganhos, e não apenas em experiências negativas (Neri, 1997).

Em síntese, sintomas depressivos na velhice podem ser influenciados por variáveis de outros períodos do curso da vida, em especial, em estágios iniciais e de transição, como a infância e a adolescência. Todavia, torna-se importante observar também outras variáveis implicadas neste processo. 


\section{Conclusão}

A partir das análises realizadas, observou-se que os estressores na infância e adolescência exerceram, para esta amostra, um efeito em longo prazo na saúde emocional, corroborando a hipótese inicial de que idosos com mais vivências estressantes na infância e adolescência apresentam mais sintomas depressivos na velhice. Contudo, suporte social na infância e adolescência, ao contrário do esperado, não demonstrou efeito protetor para sintomas depressivos na velhice.

Ressalta-se ainda a relação entre punição física e recompensas dadas pelos pais na infância e adolescência com a presença de sintomas depressivos. Enquanto as punições não tiveram uma correlação significativa com sintomas depressivos, ou seja, não estiveram relacionadas com alterações no estado emocional dos idosos, maior freqüência de recompensas, por sua vez, correlacionou-se significativamente com baixos escores de sintomas depressivos.

Entretanto, estes resultados, assim como em muitos estudos de rastreamento populacional, apresentam algumas limitações. Dentre estas, a utilização de instrumentos sem validade ecológica, já que a escala utilizada para investigar as variáveis da infância e adolescência foi apenas crosstraduzida, não sendo ainda validada no Brasil. Por fim, sugere-se que futuros estudos busquem explorar as variáveis investigadas, considerando possíveis diferenças entre gêneros e outras coortes históricas.

\section{Referências}

Aldwin, C. M., Cupertino, A. P., Levenson, M. R., \& Spiro, A., III (1998). Childhood experiences and health outcomes in later life [Abstract]. The Gerontologist, 38, 64.

Aldwin, C. M., Spiro, A., III, Levenson, M. R., \& Cupertino, A. P. F. B. (2001).

Longitudinal findings from the normative aging study III: Personality, individual health trajectories and mortality. Psychology and Aging, 16(3), 450-465.

Aldwin, C. M., Sutton, K. J., Chiara, G., \& Spiro, A., III. (1996). Age differences in stress, coping, and appraisal: Findings from the normative aging study. Journal of Gerontology: Psychological Sciences, $51 b(8) 117-188$.

Aquino, F. T. M. de, \& Cabral, B. E. S. da (2002). O idoso e a família. In E. V. de Freitas, L. Py, A. L. Neri, F. A. X. Cançado, M. L. Gorzoni, \& S. M. da Rocha (Eds.), Tratado de Geriatria e Gerontologia (pp. 1056-1060). Rio de Janeiro, RJ: Guanabara Koogan.

Blazer, D., Hughes, D., \& George, L. (1987). The epidemiology of depression in an elderly community population. The Gerontologist, 27, 281-287.

Cecconello, A. M., \& Koller, S. H. (2000) Competência social e empatia: Um estudo sobre resiliência com crianças em situação de pobreza [Versão eletrônica]. Estudos de Psicologia (Natal), 5(1), 71-93. Retirado em 20 jul. 2004, do SciELO (Scientific Electronic Library Online) em http://www.scielo.br/prc
Cupertino, A. P. (2001). Tradução da escala do inglês para o português. Estudo dos processos de envelhecimento saudável. Juiz de Fora. MG: Editora da Universidade Federal de Juiz de Fora.

Cupertino, A. P. F. B., Neri, A. L., Silva, K., Banhato, E., \& Novaes, C. (2004). Envelhecimento em uma amostra de idosos randomicamente selecionados em Juiz de Fora. Manuscrito em preparação.

Fortes, A. C. G., \& Neri, A. L. (2004). Eventos de vida e envelhecimento humano. In A.L. Neri \& M. S. Yassuda (Eds.), M. Cachioni (Colab.), Velhice bem-sucedida: Aspectos afetivos e cognitivos (pp. 51-70). Campinas, SP: Papirus.

Gazalle, F. K., Lima, M. S., Tavares, B. F., \& Hallal, P. C. (2004). Sintomas depressivos e fatores associados em população idosa no Sul do Brasil [Versão eletrônica]. Revista de Saúde Pública, 38(3), 365-371. Retirado em 01 set. 2004, do SciELO (Scientific Electronic Library Online) em http:/ / www.scielo.br/prc

Goldstein, L. L. (1995). Stress e coping na vida adulta e na velhice. In A. L. Neri (Ed.), Psicologia do envelhecimento (pp. 145158). Campinas, SP: Papirus.

Lafer, B., Almeida, O. P., Fraguas, R., Jr., \& Miguel, E. C., Filho (2001). Depressão no ciclo da vida. Porto Alegre, RS: Artes Médicas.

Levenson, M. R., Aldwin, C. M., \& Cupertino, A. P. (2001). Transcendendo o ego: O modelo liberador do desenvolvimento humano. In A. L. Neri (Ed.), Maturidade e velhice: Trajetórias individuais e socioculturais (pp. 99-115). Campinas, SP: Papirus.

Lipp, M. E. N. (Ed.). (2000). Crianças estressadas: Causas, sintomas e soluções. Campinas, SP: Papirus.

Miguel, E. C., Filho, \& Almeida, O. P. de (2000). Aspectos psiquiátricos do envelhecimento. In E. T. de C. Filho \& M. P. Netto (Eds.), Geriatria, fundamentos, clínica e terapêutica (pp. 63-82). São Paulo, SP: Atheneu.

Neri, A. L. (1997). A pesquisa em Gerontologia no Brasil: Análise de conteúdo de amostras de pesquisa em psicologia no período de 1975-1996. Texto Contexto de Enfermagem, 6(2), 69-105.

Neri, A. L. (2001). Paradigmas contemporâneos sobre o desenvolvimento humano em Psicologia e em Sociologia. In A. L. Neri (Ed.), Desenvolvimento e envelhecimento: Perspectivas biológicas, psicológicas e sociológicas (pp. 11-35). Campinas, SP Papirus.

Neri, A. L. (2002). Teorias Psicológicas do Envelhecimento. In E. V. de Freitas, L. Py, A. L. Neri, F. A. X. Cançado, M. L. Gorzoni, \& S. M. da Rocha, Tratado de Geriatria e Gerontologia (pp. 32-46). Rio de Janeiro, RJ: Guanabara Koogan.

Radloff, L. S. (1977). The CES-D scale: A self-report depression scale for research in the general population. Applied Psychological Measurement, 1, 385-401.

Rowe, J. W., \& Kahn, R. L. (1998). Successful aging. New York: Pantheon Books.

Silveira, D. X., \& Jorge, M. R. (2000). Escala de rastreamento populacional para depressão (CES-D) em populações clínica e não-clínica de adolescentes e adultos jovens. In: C. Gorestain, L. H. S. G. Andrade, \& A. W. Zuarde (Eds.), Escalas de avaliação clínica em Psiquiatria e Farmacologia (pp. 125-134). São Paulo, SP: Lemos

Snowdon, J. (2002). How high is the prevalence of depression in old age [Versão eletrônica]? Revista Brasileira de Psiquiatria, 24(1), 42-47. Retirado em 01 ago. 2004, do SciELO (Scientific Electronic Library Online) em http://www.scielo.br/prc 
Staudinger, U. M., Marsiske, M., \& Baltes, P. B. (1995). Resiliência e níveis de capacidade de reserva na velhice: Perspectivas da teoria de curso de vida. In A.L. Neri (Ed.), Psicologia do envelhecimento (pp. 195-228). Campinas, SP: Papirus.

Stoppe, A., Jr, \& Neto, M. R. L. (1999). Depressão na terceira idade: Apresentação clínica, abordagem terapêutica. São Paulo, SP: Lemos.
Tavares, S. S. (2004). Sintomas depressivos entre idosos: Relações com classe, mobilidade e suporte social percebidos e experiência de eventos estressantes. Dissertação de Mestrado não-publicada, Curso de Pós-Graduação em Gerontologia, Universidade Estadual de Campinas, SP.

Recebido: 30/11/2004

$1^{a}$ revisão: $10 / 02 / 2005$

$2^{a}$ revisão: $25 / 11 / 2005$

Aceite final: $15 / 12 / 2005$ 